\title{
Management of Severe Hypoglycemia at the End of Life in Non-Diabetic Patients: A Case Study and Recommendations
}

\author{
Faustino Gonzalez ${ }^{\mathrm{a}, \mathrm{c}}$, Ramesh Roshan ${ }^{\mathrm{a}}$, Richard Levene ${ }^{\mathrm{a}, \mathrm{b}}$, Barry Miskin ${ }^{\mathrm{a}}$, \\ Norberto Vazquez ${ }^{\mathrm{a}}$
}

\begin{abstract}
We present a case of a patient with metastatic synovial cell sarcoma of the lung with paraneoplastic production of insulin like growth factor (ILG-F) whose main symptom was generalized weakness due to severe hypoglycemia, and we also provide a brief discussion of the management of hypoglycemia at the end of life.
\end{abstract}

Keywords: Paraneoplastic, Hypoglycemia; End of life; ILG-F

\section{Introduction}

When blood glucose level falls below $70 \mathrm{mg} / \mathrm{dL}(3.9 \mathrm{mmol} / \mathrm{L})$, most patients begin to exhibit symptoms of hypoglycemia, both sympathetic (tachycardia, palpitations, diaphoresis, and tremulousness) and parasympathetic (nausea and hunger). Symptoms of neuroglycopenia can appear at blood glucose levels of $50 \mathrm{mg} / \mathrm{dL}(2.8 \mathrm{mmol} / \mathrm{L})$ and include irritability, confusion, blurred vision, tiredness, difficulty speaking, and headaches. The most severe complications of hypoglycemia include seizure, coma, and death, which can occur at blood glucose levels of $<40 \mathrm{mg} / \mathrm{dL}$ [1]. This severe hypoglycemia requires assistance of another person to actively administer carbohydrates, glucagon, or take other corrective actions [2]. Hypoglycemia is confirmed by documentation of Whipple's triad: symptoms suggesting of hypoglycemia, a low plasma glucose concentration at the time of symptoms and resolution of the symptoms after the plasma glucose level is raised [3]. Hypoglycemia may also go unrecognized in the elderly population because of restricted communication, cognitive impairment and possibly

Manuscript accepted for publication August 13, 2015

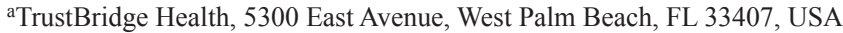
${ }^{b}$ Hospice \& Palliative Medicine Fellowship Program, University of Miami Miller School of Medicine Palm Beach Regional Campus, Atlantis, FL 33462, USA

${ }^{\mathrm{c} C o r r e s p o n d i n g ~ A u t h o r: ~ F a u s t i n o ~ G o n z a l e z, ~ T r u s t B r i d g e ~ H e a l t h, ~} 5300$ East Avenue, West Palm Beach, FL 33407, USA.

Email: fgonzalez@trustbridge.com

doi: http://dx.doi.org/10.14740/jmc2260w fewer adrenergic symptoms [4]. Risk factors for developing hypoglycemia include sepsis, a decrease in serum albumin level by $1 \mathrm{~g} / \mathrm{dL}$, malignancy, sulfonylurea, insulin treatment, alkaline phosphatase level, female gender, and elevated creatinine levels [5]. Hypoglycemia may be a paraneoplastic syndrome associated with tumors that secrete ILG-F [6].

\section{Case Report}

A 73-year-old white male presented to the emergency room via paramedics with generalized weakness. He was found to have a fasting blood sugar of $30 \mathrm{mg} / \mathrm{dL}(1.7 \mathrm{mmol} / \mathrm{L})$ by the paramedics without previous similar episodes or a history of diabetes. Additionally, the patient complained of pain limited more to the left thoracic region, significant shortness of breath even at rest with orthopnea, ongoing productive cough with clear phlegm and chronically swollen legs. Patient denied any headache, visual changes, fever or chills. He did not complain of any abdominal pain, nausea, vomiting and no change in bowel or bladder habits.

His difficulty breathing was alleviated by oxygen, nebulizer treatments and draining of the pleural fluid. He had been on oxygen at home for the past 2 weeks and had a Pleurex catheter placed in the left chest wall. He was visited by nurses at home three times a week and each time on average $500 \mathrm{~mL}$ of the pleural fluid was drained.

The patient had a history of synovial cell sarcoma treated with oral chemotherapy for the past 6 years. Unfortunately, despite treatment, the disease continued to progress. Over time patient developed a large left malignant pleural effusion and bulky tumor burden due to the synovial cell sarcoma. He had also developed significant hoarseness over a period of 3 months. Furthermore, he had a rapid deterioration over the last 4 months with regard to weight loss of 30 pounds, hypoxia and rapidly accumulating pleural effusions. As a result, the patient was referred to hospice for symptom control and end of life comfort measures.

The patient was admitted to the inpatient unit and was treated with D10W, D50, glucagon and dietary interventions. He continued to have recurring episodes of hypoglycemia despite the treatment.

Patient was on the following medications: tamsulosin 0.4 $\mathrm{mg}$ PO daily, hydrocodone $10 \mathrm{~mL}$ every $6 \mathrm{~h}$ as need for cough, 
furosemide $20 \mathrm{mg}$ every other day, potassium chloride $10 \mathrm{mEq}$ every other day, atrovastatin $60 \mathrm{mg}$, and hydrocodone/homatropine $10 \mathrm{~mL}$, also as needed for cough.

On physical examination, the patient appeared acutely ill with loose fitting clothes, cachectic with temporal wasting, and in moderate respiratory distress. His vital signs were found to be within normal limits except respiration of 20 breaths per minute. The head and neck examination found no significant JVD, anicteric sclera, and dry mucous membrane of the oropharynx. The breath sounds were diminished mostly in the left hemithorax, and on the right side with some rhonchi that improved with cough. Examination of the extremities revealed no clubbing or cyanosis. There appeared a non-tender bilateral $2+$ pretibial edema, a mildly gross motor weakness with muscular atrophy. Examination of the pulses and distal extremities revealed adequate perfusion. The cardiovascular and abdominal examinations were within normal limits.

Diagnostic studies excluded myocardial infarction, electrolyte abnormalities, renal failure and infection as the etiology of the generalized weakness. However, his blood glucose was persistently low, 20 - $80 \mathrm{mg} / \mathrm{dL}$ (1.1 - $4.4 \mathrm{mmol} / \mathrm{L})$.

CT scan of the chest without contrast revealed near complete collapse of left lung with minimal aerated portions, masslike appearance present in the left hemithorax, predominantly involving the entire left upper lobe region with a separate $3 \mathrm{~cm}$ mass in the left lower lobe. There was also a large $9 \mathrm{~cm}$ right lung paramediastinal mass with an additional $1.4 \mathrm{~cm}$ right lower lobe nodule. A subcarinal lymph node was prominent. Findings were consistent with bilateral neoplasm and could reflect primary and/or metastatic lesions. Ultrasound of the bilateral lower extremities revealed no DVT.

\section{Discussion}

Hypoglycemia at the end of life has not been widely reported in the palliative medicine literature. We did a PubMed and Google search using the following terms: "hypoglycemia at the end of life, palliation of hypoglycemia, hyperinsulinism at the end of life," and found only three previous case reports that involved the palliation of this symptom in association with ILGF producing tumors [7-9]. Hypoglycemia is an event requiring the assistance of another person to actively administer carbohydrates, glucagon, or take other corrective actions. Glucose levels may not be available during the event, but resolution of symptoms following therapeutic intervention is considered sufficient evidence that it was the result of low blood glucose concentration $[2,3]$. Whipple described a triad of low blood sugar, neuroglycopenic symptoms, and the return to baseline when glucose is administered [10]. Symptoms of hypoglycemia can range from irritability, confusion, blurred vision, difficulty speaking or tiredness, usually seen with blood sugar in the $50 \mathrm{mg} / \mathrm{dL}(2.8 \mathrm{mmol} / \mathrm{L})$, to seizures, coma or death, usually seen with glucose levels of less than $40 \mathrm{mg} / \mathrm{dL}(2.2 \mathrm{mmol} / \mathrm{L})$ [11]. The symptoms of hypoglycemia can be quite uncomfortable and lead to anticipatory fear, a phenomenon known as fear of hypoglycemia (FOH) [12]. It can also have a negative impact on family members of patients who experience it [13].

The occurrence of hypoglycemia in older non-diabetic patients is associated with increased in-hospital mortality [5]. A review of a large number of patients who visited the emergency department revealed that, in addition to patient's age, any pre-existing advanced liver disease, cancer, and coexisting sepsis, the blood glucose level was one of the strongest predictors of death in non-diabetic patients who experienced hypoglycemia [14]. Other risk factors for hypoglycemia include sepsis, a decrease in serum albumin by $1 \mathrm{~g} / \mathrm{dL}$, malignancy, female gender and elevated creatinine levels [5]. Most recently, the analgesic tramadol has been identified as a potential cause of this disorder [15].

\section{Barriers to recognizing hypoglycemia at the end of life}

In palliative and end of life care, quality of life and comfort are paramount. As a result, there is a tendency to perform less tests and finger pricks. Another barrier is that testing is associated with psychological and physical discomfort due to the fingerprick blood sampling. Additionally, there is the inconvenience of time requirements, physical setting and the complexity of the technique [16].

Hypoglycemia may also go unrecognized in the elderly population because of restricted communication, cognitive impairment and possibly fewer adrenergic symptoms [4]. Moreover, altered mental status in a non-diabetic patient is often attributed to progression of the disease. Because this derangement may go unrecognized, care providers need to be aware of its signs and symptoms and, even though finger sticks may be uncomfortable, they may be warranted in patients who are at risk, as described above $[5,16]$.

\section{Management strategies}

Treatment of hypoglycemia at the end of life should be convenient, non-invasive, consistent with the goals of care set by the patient, and should avoid disruption of the individual's care environment. Furthermore, return to consciousness may not always be desirable, especially in patients who are imminently dying or have concomitant refractory symptoms. In these cases, supportive care that addresses the symptoms without correcting the blood sugar may be preferred. The use of benzodiazepines, either intravenously or subcutaneously, may be effective in managing the associated anxiety from hypoglycemia.

In the acute setting, regardless of the etiology, boluses of D50W by intravenous administration, oral glucose paste or glucagon by intramuscular or subcutaneous route may be given. D50W works almost immediately and contains $25 \mathrm{~g}$ of dextrose in only $50 \mathrm{~mL}$ of fluid. A continuous intravenous infusion of D10W may also be used. However, this requires intravenous access which is not always possible in certain care settings. Glucagon typically will work within $5 \mathrm{~min}$. Oral glucose tablets and paste can take up to 30 min to work [6].

In patients with longer life expectancies and more chronic episodes of hypoglycemia, dexamethasone $[7,8]$ can be given orally or subcutaneously. Glucocorticoids are inexpensive but potential for short-term and long-term adverse effects has to be 
considered [17]. Human growth hormone (HGH), octreotide, diazoxide, and nifedipine have also been reported as therapeutic options. Octreotide can cause paradoxic hypoglycemia; however, it may be prudent to administer a test dose to determine if it is a therapeutic option for a particular patient [17, 18]. It should also not be used in patient with low glycogen stores. This can be determined by administering a $1 \mathrm{mg}$ intravenous glucagon stimulation test [19]. These recommendations have been summarized in fast fact format elsewhere [20].

\section{Our patient, continued}

Dexamethasone, $4 \mathrm{mg}$ by mouth daily, was added to the treatment regimen. On day 4 post-treatment, his glucose levels remained above $100 \mathrm{mg} / \mathrm{dL}(5.6 \mathrm{mmol} / \mathrm{L})$ and his symptoms stabilized. He was discharged home with hospice on day 6 . Several weeks later, he was readmitted to the inpatient unit for end of life care, as he did not want to die at home. He died peacefully without experiencing any further drops in his glucose level.

\section{Conclusion}

We presented a case of a non-diabetic patient who presented with hypoglycemia associated with an ILG-F producing tumor. Our review of the palliative literature revealed a paucity of case reports of this phenomenon [7-9] and no clear guidelines for the management of hypoglycemia in terminally ill non-diabetic patients. It is possible that based on other reports $[4,5,14,15]$, the prevalence of this metabolic derangement is greater than suspected and we set out to suggest some management strategies that will surely need further study. Additionally, because this derangement may go unrecognized, care providers need to be aware of its signs and symptoms and, even though finger sticks may be uncomfortable, they may be warranted in patients who are at risk $[5,16]$.

\section{Conflict of Interest}

The authors have no conflict of interest disclosures to report.

\section{Author Contributions}

All authors participated sufficiently in the intellectual content of this presentation.

\section{References}

1. Pasala S, Dendy JA, Chockalingam V, Meadows RY. An inpatient hypoglycemia committee: development, successful implementation, and impact on patient safety. Ochsner J. 2013;13(3):407-412.

2. Seaquist ER, Anderson J, Childs B, Cryer P, Dagogo-
Jack S, Fish L, Heller SR, et al. Hypoglycemia and diabetes: a report of a workgroup of the American Diabetes Association and the Endocrine Society. Diabetes Care. 2013;36(5):1384-1395.

3. Cryer PE, Axelrod L, Grossman AB, Heller SR, Montori VM, Seaquist ER, Service FJ. Evaluation and management of adult hypoglycemic disorders: an Endocrine Society Clinical Practice Guideline. J Clin Endocrinol Metab. 2009;94(3):709-728.

4. Kagansky N, Levy S, Rimon E, Cojocaru L, Fridman A, Ozer Z, Knobler H. Hypoglycemia as a predictor of mortality in hospitalized elderly patients. Arch Intern Med. 2003;163(15):1825-1829.

5. Abdelhafiz AH, Baily C. Sinclair A. Hypoglycemia in hospitalized non-diabetic older people. European Geriatric Medicine. 2012;3(3):174-178.

6. Pelosof LC, Gerber DE. Paraneoplastic syndromes: an approach to diagnosis and treatment. Mayo Clin Proc. 2010;85(9):838-854.

7. Scott K. Non-islet cell tumor hypoglycemia. J Pain Symptom Manage. 2009;37(4):e1-3.

8. Ying CK. Two Patients With Hypoglycaemia. Palliative Medicine Grand Rounds; HKSPM Newsletter.2008;(2):25-27.

9. Johnson T, Kumar SC. Nonislet Cell Tumor Hypoglycemia. Case Reports in Endocrinology, volume 2013. Article ID 308086, 3 pages.

10. Service FJ. Hypoglycemic disorders. N Engl J Med. 1995;332(17):1144-1152.

11. Whipple AO, Frantz VK. Adenoma of Islet Cells with Hyperinsulinism: A Review. Ann Surg. 1935;101(6):12991335.

12. Wild D, von Maltzahn R, Brohan E, Christensen T, Clauson P, Gonder-Frederick L. A critical review of the literature on fear of hypoglycemia in diabetes: Implications for diabetes management and patient education. Patient Educ Couns. 2007;68(1):10-15.

13. Gonder-Frederick L, Cox D, Kovatchev B, Julian D, Clarke W. The psychosocial impact of severe hypoglycemic episodes on spouses of patients with IDDM. Diabetes Care. 1997;20(10):1543-1546.

14. Tsujimoto T, Yamamoto-Honda R, Kajio H, Kishimoto M, Noto H, Hachiya R, Kimura A, et al. Prediction of 90-day mortality in patients without diabetes by severe hypoglycemia: blood glucose level as a novel marker of severity of underlying disease. Acta Diabetol. 2015;52(2):307314.

15. Fournier JP, Azoulay L, Yin H, Montastruc JL, Suissa S. Tramadol Use and the Risk of Hospitalization for Hypoglycemia in Patients With Noncancer Pain. JAMA Intern Med. 2015;175(2):186-193.

16. Tests of glycemia in diabetes. Diabetes Care. 2000;23;(Suppl 1):S80-82.

17. Bodnar TW, Acevedo MJ, Pietropaolo M. Management of non-islet-cell tumor hypoglycemia: a clinical review. J Clin Endocrinol Metab. 2014;99(3):713-722.

18. Vezossi D, Bennet A, Rochaix P, Courbon F, Selves J, Pradere B, Buscail L, Susini C, Caron P. Octreaotide in innsulinoma patients: efficacy on hypoglycemia, relation- 
ships with Octreoscan Scintigraphy and immunostaining with anti-sst2A and anti-sst5 antibodies. European Journal of Endocrinoly. 2005;152(5):757-767.

19. Nayar M, Lombard M, Furlong N, McNulty S, Hardy K, Vora J. Diagnosis and Management of nonislet cell tumor hypoglycemia: case series and review of the literature. The Endocrinologist;16 (4): 227-230.

20. Gonzalez F, Roshan R, Levene RS. Hypoglycemia management in nondiabetic adults at the end of life \#291. J Palliat Med. 2015;18(6):552-553. 